\title{
Antiviral and antiretroviral drug shortages amidst COVID-19: How Africa is struggling
}

Sudhan Rackimuthu ${ }^{1}$; Zohra Kazmi ${ }^{2}$; Osman Kamal Osman Elmahi3 ${ }^{3}$ Reem Hunain ${ }^{4}$; Behram Khan Ghazi ${ }^{5}$; Zain Ali Zaidi ${ }^{2}$; Ana Carla dos Santos Costa ${ }^{6}$; Shoaib Ahmad ${ }^{5}$; Mohammad Yasir Essar ${ }^{7}$

${ }^{1}$ Father Muller Medical College, Mangalore, Karnataka, India; ${ }^{2}$ Jinnah Medical and Dental College, Karachi, Pakistan;

${ }^{3}$ Faculty of Medicine, Ibn Sina University, Khartoum, Sudan; ${ }^{4}$ Kasturba Medical College, Manipal, India;

${ }^{5}$ Punjab Medical College, Faisalabad, Pakistan; ${ }^{6}$ Federal University of Bahia, Salvador, Bahia, Brazil;

${ }^{7}$ Kabul University of Medical Sciences, Kabul, Afghanistan

\section{Abstract}

Antiviral drugs are of paramount importance in the accomplishment of the vision of zero new cases of COVID-19 globally, through sustainable retaliation against viral diseases. However, several challenges currently exist in Africa which include insufficient infrastructure, deteriorating health systems, and rising costs of healthcare delivery with concomitant rising inequity with regards to access to health services amid the COVID-19 pandemic. The pandemic itself has stimulated an increased use of phytotherapy in Africa as a result of essential drug shortages that have been attributed to a plethora of contributing factors such as travel restrictions, reduced per capita income as well as increased expenditure on transport. As a result, the paucity of antiviral along with antiretroviral drugs used to combat COVID-19 as well as several other endemic viral diseases in Africa has created a worrisome state. This article therefore discusses and aims to underscore the causes, effects, and implications of antiviral and antiretroviral shortages amid COVID-19 in Africa.

\section{Introduction}

Antivirals and antiretrovirals alongside vaccines are notable developments in the field of medicine and are a vital element of well-organized, sustainable retaliation against viral diseases to accomplish the vision of zero new documented cases, zero prejudice and zero disease related deaths ${ }^{[1]}$.Antivirals appreciably aid in the world attaining the international goal of abolition of viral diseases such as hepatitis C by 2030. Hence, the challenge faced by various stakeholders and countries lies with their capability to access these treatments due to their comparatively elevated rates ${ }^{[2]}$.

Among the various problems in introducing antiviral and antiretroviral therapy in Africa, one of them is the insufficient infrastructure to deal with the number of people infected. In Africa, a large proportion of patients fall into the category of potential poor adherers unless resource intensive adherence programs are available ${ }^{[3]}$. Several drawbacks that additionally arise include the disintegration of health systems, worsening health care unfairness and objectionable opportunity expenses. In sub-Saharan Africa (SSA), millions of dollars have been utilized for this cause through governments as well as via the Global Fund, the World Bank, and bilateral development aid from abroad. Private foundations such as the Gates and Clinton Foundations, along with nongovernmental organizations like that of Médecins Sans Frontières, have provided funds and technological support, enabling the government to distribute maximum share into health and eventually increased access to antiviral drug therapy in Africa. Additionally, funds from various organizations were utilized by making the drugs cost effective so that any individual could afford antiviral drug therapy when required ${ }^{[4]}$.

Corresponding author: Osman Kamal Osman Elmahi

Faculty of Medicine, Ibn Sina University

Khartoum, Sudan

Email: osman19091995@gmail.com
Essential medicines are the backbone of healthcare requirements of any inhabitant of a particular region. The upsurge in the number of cases of COVID-19 around the world, which led to the unavoidable lockdowns as well as disruption of financial systems across affected countries, resulted in a visible reduction in manufacture and export of medicines along with other raw materials. This drastically affected the ease of access to antiviral drugs. Also, most of the developing countries are in their premature stages of pharmaceutical advancement, which indicates that they depend on importation of medicines as well as raw materials from countries outside their own. Close to $70 \%$ of the medicines used in most African countries are imported from China and India. Lockdowns were accompanied with the closure of borders and travel bans across states leading to a significant drop in the quantity of essential medicines in the health facilities, making it difficult for consumers to access the medicines.

The COVID-19 pandemic also caused an increase in the prices of medicines and other medical equipment used for providing health care ${ }^{[5]}$.There has been rising incidences in use of phytomedicines also known as herbal medicine or phytotherapy which is well accepted in multiple Pan African domains where 80 to $90 \%$ of its rural inhabitants rely on conventional treatment, which is primarily plant based for primary healthcare, due to the antiviral drug shortage during COVID-19 pandemic. The widespread use of the plant-based medicine in Africa which is also referred to as Traditional African Medicine (TAM) has been narrated to be linked with African demographic and socio-cultural funding. WHO has therefore planned to sensitize African member states toward the incorporation of TAM as well as modern medicine into their health system as the body recognizes the significance of harmonizing the two different forms of medicine as Africa has had a long history of TAM and educated native practitioners. It has been thought off as useful, reasonable, and accessible to 
the inhabitants by aiding the feeble African health sector ${ }^{[6]}$. Antiviral drugs are essential, as they aid in the prevention and treatment of multiple viral infections and their complicated sequelae, aiding in the mitigation and control of viral diseases which are widespread in Africa. The antiviral shortages will have an adverse impact on the continent, and this article therefore aims to underscore the impacts of antiviral shortages amid COVID-19 in Africa.

\section{Drug shortage crisis amidst the COVID-19 pandemic}

Proper accessibility, availability, and affordability to qualityassured medication is a key to meeting Sustainable Development Goal No. 3 and is necessitous for any rich and robust healthcare system. Throughout the African region, preexisting drug shortage crises have been drastically affected by the resultant effects of the COVID-19 pandemic on global supply chains. Reliance on economic superpowers for drug security has been an ongoing trend in African countries for many years. However, the COVID-19 pandemic worsened the already critical reliance of African countries on China and India for their drug supply.

In Rwanda, international manufacturers play an integral role in the supply of antiviral medicines. Insufficient formulations are produced locally and dependence on global drug aid is therefore crucial to achieving drug security in Rwanda. Despite facilitation efforts to establish businesses for Rwandan and foreign nationals, a wide gap exists in both investment and capacity building towards setups for drug manufacturing. As a result, essential drug supplies in Rwanda have been shortened further due to limited access ${ }^{[7]}$.

In Nigeria, approximately $70 \%$ of drugs are imported amid decreased production of local formulations. As a result of decreased production and global lockdowns due to COVID-19, quality-assured essential drugs in Nigeria have become scarce. Concomitantly, as a result there has been a high demand for standardizing herbal drugs to combat COVID-19. However, due to inadequate research support by the Nigerian government for this movement, control of the international pharmaceutical industry in the Nigerian healthcare system has culminated in unfair competition between imported drugs and local formulations ${ }^{[8]}$.

East African Community (EAC) nations also demonstrate a similar picture because of preventive measures that have been employed due to the pandemic. In Sudan, economic fragility has been historically known to have a high impact on drug security. Due to the lack of sound economic planning by the Sudanese transitional government, uncertainty and risk have left global manufacturers reluctant to invest. Furthermore, the supply chain of essential drugs in Sudan has been exacerbated by the ripple effect it has established with the increased COVID19 incidence rate. Provincial lockdowns had imposed curfews, closed land borders, and suspended public transport. An adverse effect was observed on the provision of service in community pharmacies in rural areas of Khartoum State, where antiviral drug shortage is extensive. A closed cycle thus exists, as economic instability leads to inadequate healthcare funding and back to the incapacity of improving essential drug supplies [9].

\section{Potential causative factors leading to drug shortages}

COVID-19 being a global pandemic has posed unprecedented challenges for countries worldwide and devastatingly impacted medication supply due to its hazardous effects on health market and pharmaceutical sector leading to shortage of essential medicines with supply of antiretroviral drugs being the most threatened particularly in third world countries. The Africa's pharmaceutical industry is poorly developed with decreased manufacturing, ineffective research, inefficacious storage, transportation and distribution facilities, poor infrastructure of drug administration as well as control, and lacks proper financial support including funding from government, non-government organizations (NGOs) and private companies for research and development, drug manufacture, supply, and control activities ${ }^{[10]}$. Hence, these countries rely primarily on importation of foreign pharmaceutical raw materials, finished drug products, reagents, and equipment.

China and India are world's main producers and suppliers of active pharmaceutical ingredients (APIs), key starting materials (KSMs) and finished products ${ }^{[11]}$. As these countries themselves have been struggling with the disastrous effects of COVID-19 and a slowdown in production, this may have led to reduced supply of materials to several African countries. In India, the Indians Pharmaceutical Alliance (IPA) asked government to restrict of all pharmaceutical products, APIs, and formulation to domestic consumption only ${ }^{[12]}$.

To make the matters worse, the distribution of the raw materials and other products, such as packaging materials that pharmaceutical companies need to manufacture drugs has further been handcuffed due to lockdown, border closure and shut down of sea shipment, land, and air transport across the border to halt the spread of COVID-19. Additionally, inadequate human resources in manufacturing facilities and workplaces due to lockdown and physical and social distancing has been another important factor responsible for low availability of antiretroviral drugs in this time of global health crisis. The synergistic effect of shortages of materials and workforces led to further widening of the supply-demand disparity, increased prices and accessibility to medications.

Other factors responsible for shortage of drugs include increased overhead and transport expenses, the need for an alternative source of key starting materials and active pharmaceutical ingredients in the setting of de-escalating national currency. The envisaged economic stagnation along with overall deteriorating financial status during the pandemic, apparently raised the cost of some antiretroviral treatment regimens. Additionally, the limited and reduced per capita 
income due to COVID-19 crisis at the level of the end consumer, led to loss of follow-up despite antiretrovirals being free in most of the third world countries like Nigeria. Patients commonly have to pay for registration, laboratory, and transportation amongst others. This financial constraint is an important factor for non-compliance to antiretroviral therapy. Another perturbing matter is development of resistance to antivirals and antiretrovirals as a result of loss to follow-up and stocks outs [13].

Implications of antiviral and antiretroviral drug shortages in Africa

The pharmaceutical sector has been severely impacted with the overall supply chain for drugs as well as research and development actions being abruptly disrupted ${ }^{[14]}$. In this time of crisis, the ramifications of anti-viral and anti-retroviral drug shortages in particular will most definitely have extensive implications on the health care system, health institutions and its workforce.

Shortages of drugs as well as other ancillary medical equipment have the potential to directly influence patient outcomes ${ }^{[15]}$. It has been reported that shortages are known to have increased the financial burden on patients by increasing out of pocket expenditure ${ }^{[16-17]}$ as well as the overall healthcare system, impeded patient care, and have augmented patient dissatisfaction [17] They have also more distressingly led to inferior treatment, medication errors and even resulted in patient mortality [17-18] With several viral diseases being endemic to Africa ${ }^{[19]}$ which are being increasingly reported during the COVID-19 pandemic, ${ }^{[20-21]}$ the paucity of anti-viral drugs has created a worrisome state. The shortage has given rise to the emergence of several viral diseases as appropriate and timely delivery of treatment has not been possible, leading to further spread of highly transmissible viral diseases. This could further stress the already weakened healthcare support system in the future.

With the African region being one of the most affected by HIV and AIDS (Human immunodeficiency virus and acquired immunodeficiency syndrome) infection accounting for almost two thirds of the global total of new HIV infections with 25.7 million people living with HIV as of $2018,{ }^{[22]}$ the anti-retroviral drug shortage has triggered an outcry among the HIV and AIDS community. It is believed that the current stocks of medication for HIV patients could possibly run out in just a few months, because of higher costs linked to lockdowns as well as border closures imposed due to COVID-19. This could potentially put millions of people at risk from disease progression and increased chance of HIV transmission if they are unable to receive their medications ${ }^{[23]}$.

\section{Conclusion}

The COVID-19 pandemic has severely affected global economics, with the developing and under-developed countries such as Africa bearing the brunt of a majority of the devastating consequences including that of the collapsing pharmaceutical industry and that of the healthcare sector. There are currently a multitude of co-existing factors in Africa which are an important reason for an inefficient drug supply chain which has been further stressed due to pandemic. With the burden of viral and retroviral diseases being high in Africa, the shortages of drugs used to combat them can have long lasting negative health outcomes amongst the general population. It is therefore imperative for the governments, organizations, and all other relevant stakeholders in Africa as well as around the world to take prompt steps to implement necessary actions to ensure affordable and easy access to vital antiviral as well as antiviral medications amongst others, to all those in need.

Ethical approval and consent to participate: Not applicable Consent for publication: Not applicable

Availability of data and materials: Not applicable

Competing interests: None declared among the authors

Funding: Not applicable

Authors' contributions: All authors contributed equally to the manuscript

Acknowledgements: Not applicable

\section{References}

1. Chan $\mathrm{Y}, \mathrm{Ng} \mathrm{S}$, Mehta M et al. Advanced drug delivery systems can assist in managing influenza virus infection: A hypothesis. Med Hypotheses. 2020;144:110298. doi:10.1016/j.mehy.2020.110298

2. Assefa Y, Hill P, Ulikpan A, Williams O. Access to medicines and hepatitis $C$ in Africa: can tiered pricing and voluntary licencing assure universal access, health equity and fairness?. Global Health. 2017;13(1). doi:10.1186/s12992-017-0297-6

3. McCoy D, Chopra M, Loewenson R, et al. Expanding access to antiretroviral therapy in sub-saharan Africa: avoiding the pitfalls and dangers, capitalizing on the opportunities. Am J Public Health. 2005;95(1):18-22. doi:10.2105/AJPH.2004.040121

4. Stevens W, Kaye S, Corrah T. Antiretroviral therapy in Africa. BMJ. 2004;328(7434):280-282. doi:10.1136/bmj.328.7434.280.

5. Emmanuel Awucha N, ChineloJanefrances O, Chima Meshach A, Chiamaka Henrietta J, Ibilolia Daniel A, Esther Chidiebere N. Impact of the COVID-19 Pandemic on Consumers' Access to Essential Medicines in Nigeria. Am J Trop Med Hyg. 2020;103(4):1630-1634. doi:10.4269/ajtmh.20-0838

6. Attah AF, Fagbemi AA, Olubiyi O, et al. Therapeutic Potentials of Antiviral Plants Used in Traditional African Medicine With COVID-19 in Focus: A Nigerian Perspective. Front Pharmacol. 2021; 12:596855. Published 2021 Apr 26. doi:10.3389/fphar.2021.596855 
7. Uwizeyimana T, Hashim HT, Kabakambira JD, et al. Drug supply situation in Rwanda during COVID-19: issues, efforts and challenges. J Pharm Policy Pract. 2021;14(1):12. Published 2021 Jan 20. doi:10.1186/s40545-021-00301-2

8. Faiva E, Hashim HT, Ramadhan MA, et al. Drug supply shortage in Nigeria during COVID-19: efforts and challenges. J Pharm Policy Pract. 2021;14(1):17. Published 2021 Jan 22. doi:10.1186/s40545-021-00302-1

9. Lucero-Prisno D, Elhadi Y, Modber M et al. Drug shortage crisis in Sudan in times of COVID-19. Public Health in Practice. 2020;1:100060. doi:10.1016/j.puhip.2020.100060

10. Newton PN, Bond KC; 53 signatories from 20 countries. COVID-19 and risks to the supply and quality of tests, drugs, and vaccines [published correction appears in Lancet Glob Health. 2020 Jul;8(7):e900]. Lancet Glob Health. 2020;8(6):e754-e755. doi:10.1016/S2214109x(20)30136-4

11. The world needs pharmaceuticals from China and India to beat coronavirus - https://theconversation.com/theworld-needs-pharmaceuticals-from-china-and-india-tobeat-coronavirus-138388

12. Ayati N, Saiyarsarai $P$, Nikfar S. Short and long term impacts of COVID-19 on the pharmaceutical sector. Daru. 2020;28(2):799-805. doi:10.1007/s40199-020-00358-5

13. Dada DA, Aku E, David KB. COVID-19 pandemic and antiretrovirals (ARV) availability in Nigeria: recommendations to prevent shortages. Pan Afr Med J. 2020;35(Suppl 2):149. Published 2020 Aug 24. doi:10.11604/pamj.supp.2020.35.149.25639

14. How to develop and implement a national drug policy. 2nd ed. Geneva: World Health Organization; 2001. Available at: http://apps.who.int/iris/bitstream/handle/10665/42423/ 924154547X.pdf?sequence=1. Accessed July 3, 2021.

15. Phuong J, Penm J, Chaar B, Oldfield L, Moles R. The impacts of medication shortages on patient outcomes: $A$ scoping review. PLoS One. 2019;14(5):e0215837. doi:10.1371/journal.pone.0215837
16. Souliotis K, Papageorgiou M, Politi A, loakeimidis D, Sidiropoulos $\mathrm{P}$. Barriers to accessing biologic treatment for rheumatoid arthritis in Greece: the unseen impact of the fiscal crisis--the Health Outcomes Patient Environment (HOPE) study. Rheumatol Int. 2014;34(1):2533. doi:10.1007/s00296-013-2866-1

17. McLaughlin M, Kotis D, Thomson K, et al. Effects on patient care caused by drug shortages: a survey. J Manag Care Pharm. 2013;19(9):783-788. doi:10.18553/jmcp.2013.19.9.783

18. Rider A, Templet D, Daley M, Shuman C, Smith L. Clinical Dilemmas and a Review of Strategies to Manage Drug Shortages. J Pharm Pract. 2013;26(3):183-191. doi:10.1177/0897190013482332

19. Chauhan RP, Dessie ZG, Noreddin A, El Zowalaty ME. Systematic Review of Important Viral Diseases in Africa in Light of the 'One Health' Concept. Pathogens. 2020;9(4):301. Published 2020 Apr 20. doi:10.3390/pathogens9040301

20. WEEKLY BULLETIN ON OUTBREAKS AND OTHER EMERGENCIES. WHO Regional office for Africa Health emergency programme. March 7, 2021.

Available at: https://apps.who.int/iris/bitstream/handle/10665/34001 2/OEW10-0107032021.pdf. Accessed July 3, 2021.

21. WEEKLY BULLETIN ON OUTBREAKS AND OTHER EMERGENCIES. WHO Regional office for Africa Health emergency programme. February 14, 2021. Available at: https://apps.who.int/iris/bitstream/handle/10665/33970 1/OEW07-0814022021.pdf. Accessed July 3, 2021.

22. HIV/AIDS. WHO Regional office for Africa. Available at: https://www.afro.who.int/health-topics/hivaids. Accessed July 3, 2021.

23. Countries urged to act over potential HIV drug shortages, within next two months. UN News. June 22, 2021. Available at: https://news.un.org/en/story/2020/06/1066782. Accessed July 3, 2021. 